\title{
Sex-specific sleep patterns among university students in Lebanon: impact on depression and academic performance
}

This article was published in the following Dove Press journal:

Nature and Science of Sleep

17 June 2016

Number of times this article has been viewed

\author{
Colette S Kabrita' \\ Theresa A Hajjar-Muça ${ }^{2}$ \\ 'Department of Sciences, \\ ${ }^{2}$ Department of Mathematics and \\ Statistics, Faculty of Natural and \\ Applied Sciences, Notre Dame \\ University - Louaize, Zouk Mosbeh, \\ Lebanon
}

\begin{abstract}
Good sleep quality and quantity are fundamental to the maintenance of normal physiological processes. Changes in sleep patterns are commonly observed among young adults and are shown to impact neurocognitive, academic, and psychological well-being. Given the scarcity of sleep information about Lebanon and acknowledging the sex differences in various sleep dimensions, we conducted a study that aimed at assessing sex differences in sleep habits among university students in Lebanon in relation to psychoacademic status. A total of 540 students (50.6\% females) completed a questionnaire that inquired about sociodemographics and evaluated sleep quality and depression using the Pittsburg Sleep Quality Index (PSQI) and Center for Epidemiological Studies Depression Scale (CES-D), respectively. The mean PSQI global score (6.57 \pm 3.49$)$ indicated poor sleep, with no significant differences between men and women. The sleep/wake rhythm was delayed on weekends for both sexes. Females exhibited earlier bedtimes and rise times and longer sleep durations on both weekdays and weekends. However, unlike males females showed a greater phase delay in wake times than bedtimes on weekends ( 149 minutes vs 74 minutes, respectively). In all, $70.9 \%$ of females suffered from depressive symptoms, which was a significantly higher proportion compared with $58.5 \%$ of males $(P<0.01)$. Based on the mean cumulative self-reported grade point average (GPA), the academic performance of females was significantly better than that of males ( $2.8 \pm 0.61$ vs $2.65 \pm 0.61, P<0.05$, respectively). Depression, as scored by CES-D, in females was significantly negatively correlated with the cumulative GPA ( $r=-0.278, P<0.01)$, earlier wake time $(r=-0.168, P<0.05)$, and average sleep duration $(r=-0.221$, $P<0.01)$ on weekdays. GPA of males was significantly correlated with bedtime on weekends $(r=-0.159, P<0.05)$. We conclude that sex differences in sleep timing, such as bedtime/rise time and nocturnal sleep duration, rather than sleep quality exist among Lebanese university students. Sex-specific sleep patterns have differential impact on psychological and academic well-being. Keywords: bedtime-rise time, CES-D, grade point average, PSQI, young adults
\end{abstract}

\section{Introduction}

Sleep is a biological necessity that is fundamental to the maintenance of normal body processes, such that impairment has been shown to impact neurocognitive, academic, and psychological well-being, among others. ${ }^{1-3}$ Insufficient sleep, whether manifested as alteration in sleep quantity and/or quality, has been reported in various countries and is commonly noted among adolescents and young adults. ${ }^{4}$ In this age group, delayed bedtimes are common and, when rise time is determined by the school start time, short sleep duration on weekdays results. This is also often associated with extended sleep on weekends, when there are fewer morning social and academic requirements. ${ }^{5,6}$
Correspondence: Colette S Kabrita Department of Sciences, Faculty of Natural and Applied Sciences, Notre Dame University - Louaize, PO Box 72 , Zouk Mikael, Zouk Mosbeh, Lebanon

Tel +96I 9208728

Fax +96| $921877 \mid$

Email ckabrita@ndu.edu.lb 
This extended recovery sleep on weekends is a homeostatic compensatory mechanism for the sleep debt created on weekdays, although it is likely that the weekend sleep extension is insufficient to "pay back" the lost weekday sleep. In addition, the irregular bed and wake times resulting from such a schedule produce disrupted circadian rhythmicity, which can independently impact physiological, psychological, and academic well-being. ${ }^{7}$

The fundamental role of good sleep quality and quantity in information processing, learning, and memory consolidation is well established in the literature..$^{8-11}$ The impact of sleep pattern changes on academic performance has been widely investigated in young adult groups, including university students. In one study, Singleton and Wolfson ${ }^{12}$ showed the difference in bedtime habits between weekdays and weekends of college students to be directly correlated with their grade point average (GPA). Students with late sleep schedules (weekday-weekend bedtime delays) suffered more daytime sleepiness that was a strong predictor of poorer GPA. Kelly et $\mathrm{al}^{13}$ also investigated the relationship between sleep quality and GPA of undergraduate university students and found that short sleepers (total sleep time $\leq 6$ hours per night) had significantly lower GPAs than long sleepers (total sleep time $\geq 9$ hours per night). The poor academic performance associated with short sleep durations was linked to reduced concentration ability on educational material, lower selfefficacy, and possible psychological difficulties. Thus, sleep disturbance, such as alteration in the sleep/wake rhythm or nocturnal sleep duration, appears to have adverse implications on academic achievement.

As well as age, sex differences in sleep patterns have been well documented in different cultures. ${ }^{14-17}$ Females reportedly suffer more sleep dissatisfaction with increasing age relative to males. However, research findings regarding sex differences in sleep dimensions are inconsistent. Discrepancies may be explained by cultural differences in the age groups considered in the studies. According to some studies on young adult sleep patterns, females were reported to have longer mean total sleep time and poorer sleep quality compared with their age-matched male counterparts. ${ }^{18-20}$ However, other studies reported no sex differences with respect to total sleep time and the number of nocturnal awakenings in the same age group. They also found that the sleep/wake rhythm appeared to vary between men and women, with earlier timings (ie, earlier bedtimes and rise times) in young adult women. ${ }^{17}$

A number of factors appear to differentially interact in men and women as to influence sleep health, one such determinant being psychological, namely the development of depressive disorders. ${ }^{21}$ Epidemiological studies show that women, starting from adolescence throughout to adulthood, are more vulnerable and prone to depressive disorders than men. ${ }^{22-24}$ Despite the importance of this area of research, very few studies regarding sex differences in depression and overall mental health have been conducted in the Arab world, particularly in the Middle East region, where socioeconomic pressures and political conflicts are stringent. In one study, Tanios et $\mathrm{al}^{25}$ showed sex differences in the prevalence of anxiety disorders, being more common among females. Another study in the Palestinian Authority showed that female adolescents exhibited higher levels of psychological symptoms than age-matched males living under the same sociopolitical conditions. ${ }^{26}$ According to the national health reports, ${ }^{27}$ depression is an alarming public health issue in the postwar Lebanon, yet epidemiological age-based studies on the prevalence of depression among men and women and its potential risk factors are scarce. Karam et $\mathrm{al}^{28}$ investigated the lifetime prevalence of mental disorders in Lebanon and concluded that females had a higher risk of developing anxiety and mood disorders than males. Mental health among young adults is of special concern because of its impact on sleep patterns, the reverse being true as well which, in turn, affects many aspects of human life, in addition to its socioeconomic implications.

Extensive investigation of the biological, psychological, and academic significance of adequate sleep has been conducted worldwide. However, none of this work has been done in Lebanon, and very few comprehensive studies exist in the Arab Middle Eastern regions. In one study on Palestinian university students, sex differences in sleep patterns and psychological health, among other related influences, were investigated. ${ }^{29}$ However, the study was confined to a small student sample taken from one university and exhibiting sociocultural homogeneity. Furthermore, the study did not comprehensively look into variations in student sleep patterns between weekdays and weekends, and what aspects of the sleep dimensions may have specific implications on the academic performance and psychological health of males and females. As for Lebanon, the only sleep data available appear in a recent study conducted by us, ${ }^{30}$ in which we showed that sleep disturbance was prevalent among Lebanese university students, manifested as reduced sleep duration and shifts in sleep timing between weekdays and weekends. In that study, chronotype interacted with the schedules of students to affect their sleep quality and duration. Although sleep duration did not vary among the different chronotypes on weekdays (when class start times and academic demands had to be observed by students), it was both prolonged and more variable among 
chronotypes on weekends, especially for evening-type subjects. Given the scarcity of sleep information about Lebanon, as well as the sociocultural and ethnic diversity among Arab Middle Eastern regions, and to further understand the implications of poor sleep quality on psychological and academic status, we conducted the present analysis on additional data collected in that study.

Therefore, the present analysis examined data from a study of students attending different universities in Lebanon to test the hypothesis that sex differences in sleep patterns exist in this age group and that sleep/wake timing and sleep duration may interact differently in males and females to distinctly affect academic performance and psychological health.

\section{Methods}

\section{Participants and procedures}

This study was conducted at six private and public universities in different regions in Lebanon in order to secure cultural diversity, both regional and religious. To ensure a representative cross-section of students with regard to age, individual's sex, major, and level of education, up to eight sections of an undergraduate English general requirement course, hosting an average of 25 students each, all of whom were fluent in English, were selected from each university for data collection.

Prior to data collection, questionnaires were sent to each of the universities to be reviewed and approved by the local ethics board for research on human subjects (or other concerned authorities) to assure compliance with international guidelines. Data collection was done following the consent of these institutional authorities which required no written consent to the questionnaire from participating students.

For any given university, sampling was done either during the mid-fall or early spring semester of academic year 2010-2011. With the help of two trained field researchers and the respective course instructors, full-time students were orally briefed on the purpose and importance of the study during a class session and were encouraged (but not required) to complete the questionnaire. Inclusion criteria included undergraduate students in the age range of 17-25 years. Participation in the study was voluntary and did not involve financial or other compensation or course credit.

The total number of valid respondents was $540,50.6 \%$ of whom were females.

\section{Study instrument}

A pretested questionnaire (original English version) was used to collect demographic (age, individual's sex, and employment), academic (education level, GPA), and occupational and socioeconomic (employment status, perceived socioeconomic level, or household income) data. The questionnaire also included questions about specific health behaviors (such as sleep habits), overall personal health, and standardized tests. The latter part included the Pittsburgh Sleep Quality Index (PSQI) ${ }^{31}$ to assess sleep quality and related parameters (eg, sleep duration) and the Center for Epidemiological Studies Depression Scale (CES-D) to evaluate the incidence of depression.

\section{Statistical analysis and questionnaire scoring}

Data were entered, edited, and analyzed using the Statistical Package for Social Sciences Version 11.5 for windows (SPSS Inc., Chicago, IL, USA). Descriptive statistics were used to describe the demographic characteristics of the sample and to present the prevalence of behavioral habits, health conditions, sleep disorders, and quality.

Independent sample $t$-test was used for sex difference comparison of demographics, reported GPA, sleep duration, and sleep-wake habits on weekdays and weekends, in addition to comparing reported GPA between males and females for every CES-D group. One-way analysis of variance was used to compare the mean reported GPA scores among the three CES-D categories in males and females separately. Bivariate correlation tests were done to study correlations among both sexes between CES-D and GPA, sleep duration, and sleep-wake timing. Chi-square test was used for the crosstabs of individual sex with CES-D groups.

A PSQI global score $>5$ was used to categorize poor sleep. ${ }^{31}$ Based on CES-D scores, respondents were grouped into the following three categories: those with no overt signs of depression (scores <14), those with mild-to-moderate depressive symptoms (scores 15-21), and those with major depression (scores >21). ${ }^{32,33}$ As for academic performance, it was based on self-reported GPA on a scale of $0.0-4.0$, in which 0.0 is failure and 4.0 is excellent/outstanding performance.

\section{Results \\ Demographic and socioeconomic characteristics}

A total of 440 students completed the questionnaires. Their average age was $19.85 \pm 1.51$ years. Approximately half were females $(50.6 \%)$, sophomores $(53.4 \%)$, and the majority were Lebanese and/or of Lebanese descent (95.7\%). Table 1 depicts the demographic and socioeconomic characteristics of male and female student participants. 
No significant difference was observed in marital status between males and females, in which $97.7 \%$ of males and $93.8 \%$ of females were single. Approximately a third of respondents (31.6\%) reported having a job, $74.3 \%$ of who worked part-time and employment being significantly higher in males than females ( $39 \%$ vs $24.5 \%, P<0.01)$. More males reported having part-time jobs than females $(27.9 \%$ vs $18.8 \%, P<0.01)$.

Of the 509 who responded to the household income question, the majority $(79.0 \%)$ had medium income and $11 \%$ had low socioeconomic status. No significant sex differences in socioeconomic status were observed.

\section{Sex differences in sleep quality and related variables}

The mean PSQI global score of respondents was 6.57 \pm 3.49 , indicating the existence of poor sleep quality among students. Of the 218 who fully responded to the PSQI questionnaire, 58.7\% exhibited poor sleep with no significant sex differences observed in global PSQI score. Table 2 summarizes sex differences in various sleep parameters on both weekdays and weekends.

Compared with males, females had significantly earlier bedtimes on weekdays (23:53 $\pm 0: 78$ minutes vs 00:27 $\pm 0: 84$ minutes, $P<0.01)$ and weekends $(1: 06 \pm 0: 92$ hours vs 2:13 $\pm 0: 97$ minutes, $P<0.01)$. Females also had significantly

Table I Demographic and socioeconomic characteristics of male and female respondents

\begin{tabular}{|c|c|c|c|}
\hline \multirow[t]{2}{*}{ Characteristics } & \multicolumn{2}{|l|}{ Sex } & \multirow[t]{2}{*}{$P$} \\
\hline & Males & Females & \\
\hline Age, mean $\pm S D$ (years) & $20.02 \pm 1.61$ & $19.68 \pm 1.39$ & $<0.01$ \\
\hline $\mathrm{n}$ & 266 & 273 & \\
\hline \multicolumn{4}{|l|}{ Employment, (\%) n } \\
\hline Yes & $39(103)$ & $24.5(67)$ & $<0.01$ \\
\hline No & $61(16 I)$ & $75.5(206)$ & \\
\hline \multicolumn{4}{|l|}{ Household income, (\%) n } \\
\hline Low & $10.1(25)$ & II.9(3I) & NS \\
\hline Medium & $76.9(190)$ & $81.2(2 \mid 2)$ & \\
\hline High & $13(32)$ & $6.9(18)$ & \\
\hline
\end{tabular}

Notes: Independent sample t-test was used to compare mean ages between males and females; chi-square test was used to compare employment and household income between the two sexes.

Abbreviations: SD, standard deviation; NS, no significance. earlier rise times on weekdays $(7: 39 \pm 0: 83$ minutes vs 8:06 $\pm 0: 87$ minutes, $P<0.01$ ). Although the sleep/wake rhythm in both sexes shifted later on weekends than weekdays, the magnitude of the bedtime and rise time phase delay on weekends was comparable in males (106 minutes and 140 minutes, respectively) but not in females (74 minutes vs 149 minutes, respectively).

Both male and female students slept longer on weekends than on weekdays, and the women slept significantly longer than the men on weekends $(9.02 \pm 1.55$ minutes vs $8.22 \pm 1.91$ minutes, $P<0.01)$.

\section{Sex comparisons in CES-D and GPA scores}

Out of the 455 (84.3\%) who fully responded to the CES-D, $34.9 \%$ of respondents had no overt signs of depression, $24.2 \%$ were categorized as mild-to-moderately depressed, and $40.9 \%$ scored in the range of major depression. To assess sex differences in depression, the last two CES-D groups (ie, mild-to-moderate and possibility of major depression) were combined into a single group (exhibiting depressive symptoms) for comparison with those who had no signs of depression. Significant sex differences were observed in the incidence of depression, with a higher percentage of females suffering from depressive symptoms than males $(70.9 \%$ vs $58.5 \%, P<0.01)$. The results are shown in Table 3.

As shown in Table 3, the academic performance of females was significantly better than that of males based on the mean cumulative self-reported GPA $(2.8 \pm 0.61$ vs $2.65 \pm 0.61$, respectively; $P<0.05$ ). Furthermore, the mean

Table 3 CES-D and GPA scores in males and females

\begin{tabular}{llll}
\hline CES-D & Males & Females & $\boldsymbol{P}$ \\
\hline No overt depression (\%) (n) & $41.5(90)$ & $29.1(69)$ & $<0.01$ \\
Depressive symptoms (\%) (n) & $58.5(127)$ & $70.9(168)$ & \\
GPA: score/4, mean \pm SD (n) & $2.65 \pm 0.61$ (18I) & $2.80 \pm 0.61(156)$ & $<0.05$ \\
\hline
\end{tabular}

Notes: Chi-square test was used to compare CES-D categories between males and females; independent sample t-test was used to compare mean GPA score between the two sexes.

Abbreviations: CES-D, Center for Epidemiological Studies Depression Scale; GPA, grade point average; SD, standard deviation.

Table 2 Sleep variables on weekdays and weekends in males and females

\begin{tabular}{|c|c|c|c|c|c|}
\hline \multirow[t]{2}{*}{ Sleep variable } & \multicolumn{2}{|l|}{ Males } & \multicolumn{2}{|l|}{ Females } & \multirow[t]{2}{*}{$\mathbf{P}$} \\
\hline & Weekdays & Weekends & Weekdays & Weekends & \\
\hline Bedtime, mean $\pm \min (n)$ & $00: 27 \pm 84^{*}(257)$ & $2: 13 \pm 97 * *(257)$ & $23: 53 \pm 78^{*}(267)$ & $\mathrm{I}: 06 \pm 92 * *(264)$ & $<0.01$ \\
\hline Wake time, mean \pm min, $(n)$ & $8: 06 \pm 87 *(260)$ & $10: 26 \pm 1 \mid 16(26 I)$ & $7: 39 \pm 83^{*}(257)$ & $10: 08 \pm 109(266)$ & $<0.01$ \\
\hline Sleep duration, $\mathrm{h} \pm \min (\mathrm{n})$ & $7.64 \pm 1.48(253)$ & $8.22 \pm 1.91 * *(255)$ & $7.7 I \pm I .44(254)$ & $9.02 \pm 1.55 * *(260)$ & $<0.01$ \\
\hline
\end{tabular}


GPA score among the three CES-D categories was significantly different in females (one-way analysis of variance: $\left.F_{(2,140)}=5.85, P<0.01\right)$ but not in males.

The relationship between psychological well-being and academic performance was further investigated by examining the correlation between CES-D and GPA for the entire group, as well as for men and women separately. There was a significant negative correlation between CES-D and GPA in the overall group ( $r=-0.135, P=0.021)$ and, when each male and female group was examined separately, the relationship was significant in females $(r=-0.278, P<0.01)$ but not in males $(r=-0.04, P=0.625$; Figure 1$)$. Using a student's $t$-test, we further looked at sex differences in GPA within each CES-D category and found a difference only in

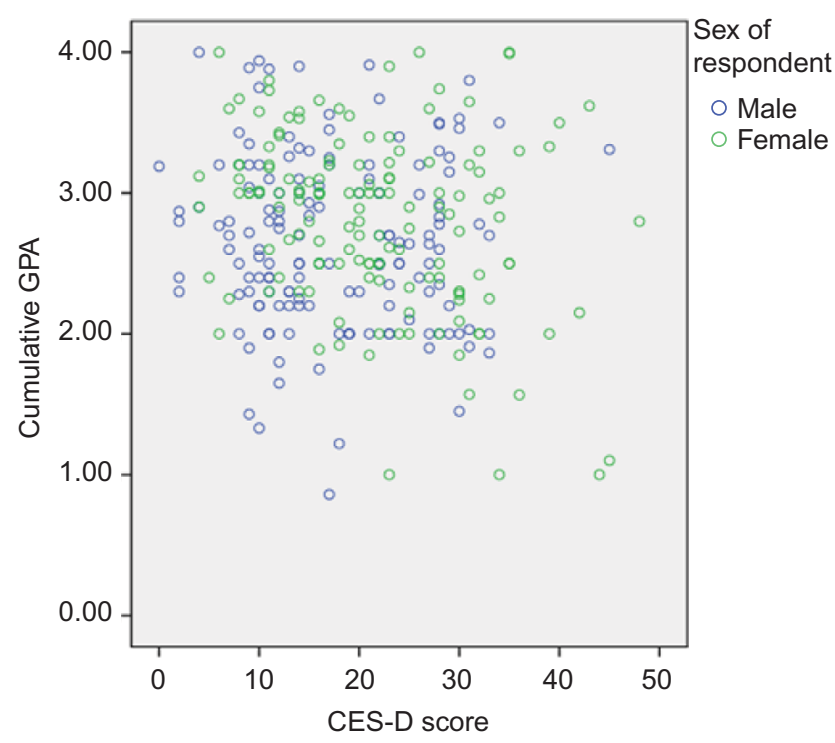

Figure I Scatterplot presenting CES-D and cumulative GPA by sex.

Notes: There was a significant negative correlation between CES-D scores and average GPA in females $(r=-0.278, P<0.01)$ but not in males, with lower GPA scores correlating with higher CES-D scores (higher incidence of depression).

Abbreviations: CES-D, Center for Epidemiological Studies Depression Scale; GPA, grade point average.

Table 4 Comparison of mean GPA among the three CES-D groups in males and females

\begin{tabular}{llll}
\hline CES-D category & Males & Females & $\boldsymbol{P}$ \\
\hline No overt depression & $2.70 \pm 0.59(69)$ & $3.07 \pm 0.47(39)$ & $<0.0 \mathrm{I}$ \\
$\begin{array}{l}\text { Mild-to-moderate } \\
\text { depression }\end{array}$ & $2.60 \pm 0.73(27)$ & $2.8 \mathrm{I} \pm 0.47(36)$ & $\mathrm{NS}$ \\
$\begin{array}{l}\text { Possibility of major } \\
\text { depression }\end{array}$ & $2.65 \pm 0.56(54)$ & $2.65 \pm 0.72(68)$ & NS \\
\hline
\end{tabular}

Notes: Values are expressed as mean \pm SD (n). P-values for Student's $t$-test denoting sex comparisons in a given CES-D category.

Abbreviations: CES-D, Center for Epidemiological Studies Depression Scale; GPA, grade point average; SD, standard deviation; NS, not significant. the group without any signs of depression, with the women having a significantly higher GPA $(3.07 \pm 0.47$ vs $2.70 \pm 0.59$, $P<0.01$; Table 4).

\section{Relationship between sleep/wake rhythm, sleep duration, cumulative GPA, and CES-D in males and females}

In females, wakeup time on weekdays, but not weekends, was negatively correlated with CES-D score $(r=-0.168, P<0.05)$, as was the average weekday sleep duration $(r=-0.221$, $P<0.01)$. No significant correlation between these sleep parameters and CES-D was observed in males. Furthermore, we observed a significant negative correlation between CES-D score and cumulative GPA in females $(r=-0.278$, $P<0.01)$ but not in males. In contrast, males' academic performance was significantly correlated with the time they went to bed on weekends ( $r=-0.159, P<0.05)$ but not on weekdays (Figure 2). Later bedtimes on weekends were associated with lower cumulative GPA scores in males but not in females.

\section{Discussion}

Sex differences in sleep quality, ${ }^{17,20}$ psychological wellbeing, ${ }^{22-24}$ and academic performance ${ }^{12}$ among university students are well established. To our knowledge, however, no research has been carried out in Lebanon to assess sleep patterns and their relationship with psychological health and academic performance among young adults. Furthermore, very few comprehensive studies on this subject exist in the neighboring Arab regions.

The current data are the first to provide such evidence on a sample of Lebanese university students in a culture-specific context. Consistent with other findings, poor sleep quality was prevalent in our student sample at a rate of $58.7 \%{ }^{34,35}$ However, This proportion of poor sleepers was more than what Sweileh et $\mathrm{al}^{36}$ reported in a study on Palestinian university students, who also showed that females had poorer sleep quality than males. ${ }^{29}$ However, this study found no sex differences in sleep quality among Lebanese university students. The differences in both findings can be reasoned, in part, to the larger sample size and broader cultural diversity (ie, inclusion of different universities across Lebanon representing different sociocultural backgrounds) in our study. Although poor sleep appears to be quite common among university students, the reported variations between different studies may be influenced by different socioeconomic demands and cultural habits among the different population groups. ${ }^{37}$

When we examined our findings by individual sex, we found significant differences in sleep habits and durations 

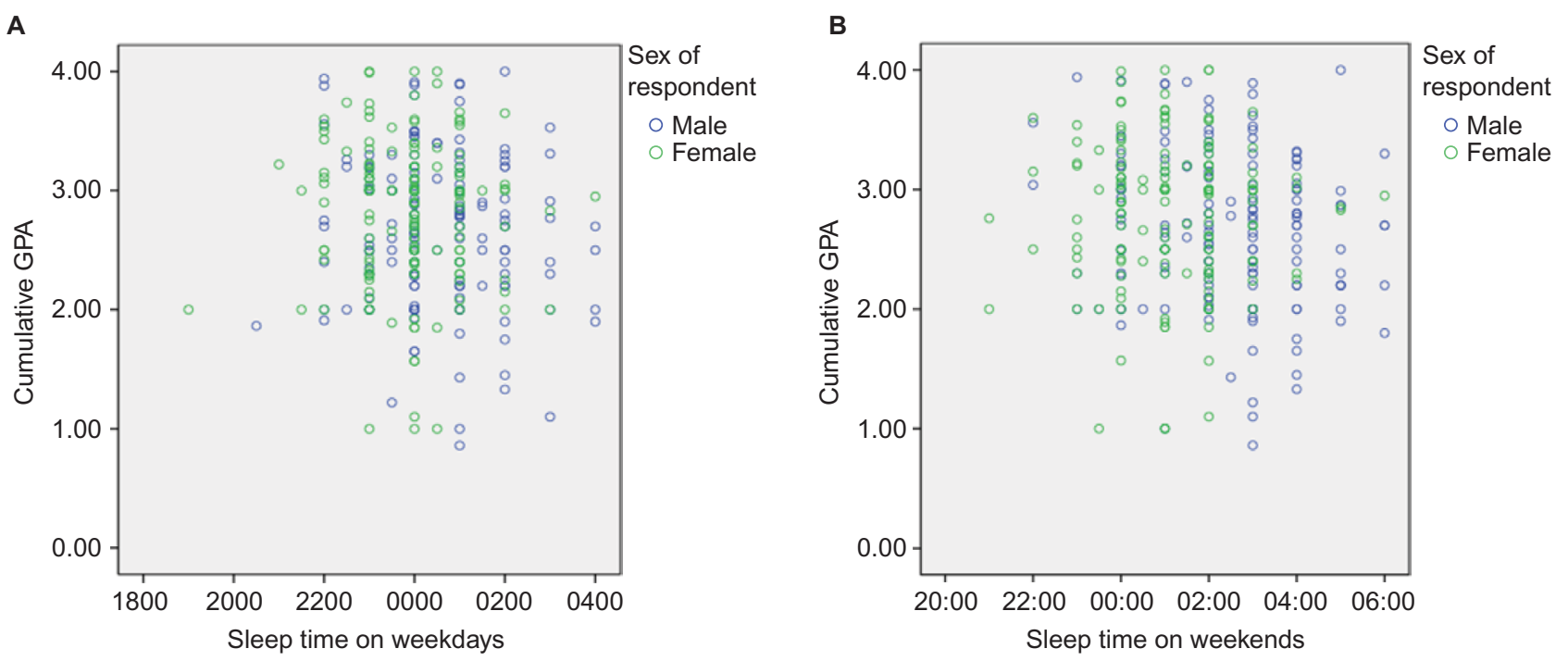

Figure 2 Scatter plot presenting sleep timing and cumulative GPA by sex on both weekdays (A) and weekends (B).

Notes: Sleep time is the hour according to the 24 hour clock. There was a significant negative correlation between bedtime and GPA in males $(r=-0.159, P<0.05)$ on weekends but not weekdays. This indicated that later sleep times on weekends were significantly correlated with poorer academic performance in males.

Abbreviation: GPA, grade point average.

between weekdays and weekends. Males had significantly later bedtimes than females on both weekdays and weekends, whereas females exhibited a significantly earlier rise time on the weekdays only. These observations are consistent with those reported by others in Arab, ${ }^{29}$ Portuguese,,${ }^{15}$ and Taiwanese $^{38}$ university students falling into the same age group as students in our study (ie, 17-25 years old). In all these studies, females generally went to bed earlier and rose earlier, slept longer, and had poorer sleep quality than male students. In the previous studies, there were no significant weekday-weekend differences between the two sexes. However, in our study the magnitude of the weekday-weekend delay in bedtime and rise time was comparable in males but not in the females, whose sleep appeared to be possibly truncated on the weekdays. In females, the amount of phase delay in bedtime mismatched the relatively more extended delay in wake time on the weekends. It is possible that the females in our study woke up at earlier times (than their habitual or biological need) on weekdays and then compensated for this on the weekends, which may also explain their significantly longer sleep duration on the weekends. Truncated sleep and alteration in wake time have substantial influence on neuroendocrine physiology by affecting the timing of the human circadian clock. ${ }^{39}$ This, in turn, may have important implications for neurocognitive function and psychological well-being.

Previous studies have found sex differences in total sleep time, with females exhibiting more sleep time than males across the life course and have attributed such differences to the different social roles and rates of paid employment of women..$^{40}$ In this study, we found a sex difference in sleep duration on weekends but not on weekdays. Although social role, particularly marital status, is unlikely to explain this sex difference in total sleep time (because reported family related obligations were comparable between male and female students), the potential contribution of paid work cannot be excluded. More male students reported paid employment, mainly on a part-time basis, than females. It is possible that male students were unable to extend their weekend sleep to the same extent as female students due to their work demands; alternatively, it may be the case that the female students need more sleep and, therefore, prioritized their free time on weekends sleeping.

Sex differences in the risk of depression and academic performance were also observed. We found a significantly higher proportion of female students exhibiting signs of depression than males. Similarly, other studies found a trend for higher lifetime rates of depression among females, especially during adolescence and adulthood. ${ }^{41}$ This trend was attributed to sex differences on exposure to socioeconomic pressures and cultural norms, reaction, and coping mechanisms to life stresses, in addition to biological (hormonal) determinants. ${ }^{24}$

As for academic performance, the average GPA score was higher in females than males. Several factors appear to contribute to the observed male-female inequality in educational achievement, including socioeconomic, demographic, psychological, and biological factors. ${ }^{42-44}$ According to this study, there was a significant negative correlation between CES-D scores and average GPA, indicating that academic 
performance declines with increased risk of depression (or depression increases with poorer academic performance). Interestingly, psychological well-being appeared to be an important determinant of good academic performance in females, but not males, as manifested by the significantly lower GPA scores among the female groups suffering various degrees of depressive symptoms. This may point to the higher susceptibility of females to the adverse effects of psychological disturbance on their academic performance. Since CES-D scores were higher and more variable in females, it is reasonable that any correlation with CES-D score will only be found in females and not in males.

To further understand how sleep, and what sleep dimension, interacts with the individual's sex to shape psychological well-being and academic performance, we investigated the relationship between the various sleep parameters, CES-D scores, and average GPA scores in both male and female students. Unlike males, psychological health in females appeared to be mainly influenced by rise times and sleep duration on weekdays. Female students were at increased risk of developing depressive symptoms with earlier rise times and shorter sleep durations on the weekdays. Furthermore, a male-female difference was also observed in the relationship between CES-D and GPA scores: lower GPA scores correlated with higher incidence of depression in females but not in males. From this, it appears that academic performance of females declines with psychological disturbance. In contrast, academic performance of males was significantly correlated with the time they went to bed on weekends but not weekdays. Later bedtimes on weekends were associated with lower cumulative GPA scores in males but not in females. Taken together, it appears that the sleep/wake rhythm may interact differently in males and females as to affect academic performance. The differential impact of the sleep/wake rhythm in the context of sex differences in the susceptibility to depression and educational inequality warrants attention and further investigation.

\section{Conclusion}

Although poor sleep quality is prevalent among a group of Lebanese university students, significant sex differences were not observed. Sex differences in sleep dimensions were noted between weekdays and weekends, in which females had earlier rise times and longer sleep on weekends, whereas males had later bedtimes on weekends. From our results, it appears that the sleep/wake rhythm may interact differently in males and females as to affect academic performance. Unlike males, the earlier rise time and shorter sleep duration in females on weekdays correlated with lower GPA scores and higher incidence of developing depressive symptoms. On the other hand, academic achievement of males appeared to be rather influenced by the time they went to bed on the weekends, with later bedtimes favoring lower GPA scores. Thus, wake up time and sleep duration may prove to be important sleep dimensions with sex-specific impact on psychological and academic well-being. Gaining insight into the mechanisms by which sleep interacts with individual sex to affect normal physiology and behavior has valuable implications in medicine and on the improvement in health-care systems.

\section{Study limitations}

Our results were based on retrospective self-reporting by students, which could be subject to bias. The use of prospective measures, such as a sleep/work/health diary, and/or objective means of documenting sleep behaviors, such as actigraphy and polysomnography recordings, may help elucidate these preliminary observations. In addition, the number of respondents to CES-D and GPA was small compared with that of the PSQI and sleep-related questions.

\section{Acknowledgments}

This study was supported by the Lebanese National Council for Scientific Research (CNRS-REF268). The authors thank Fr/ Dr Bechara Khoury and Dr Jacqueline Doumit for their input in the early stages of drafting the proposal, Ms Frida Torosyan for data entry, and Dr Jeanne Duffy for advice on the analysis.

\section{Disclosure}

The authors report no conflicts of interest in this work.

\section{References}

1. Curcio G, Ferrara M, De Gennaro L. Sleep loss, learning capacity and academic performance. Sleep Med Rev. 2006;10(5):323-337.

2. Dewald JF, Meijer AM, Oort FJ, Kerkhof GA, Bogels SM. The influence of sleep quality, sleep duration and sleepiness on school performance in children and adolescents: a meta-analytic review. Sleep Med Rev. 2010;14(3):179-189.

3. Durmer JS, Dinges DF. Neurocognitive consequences of sleep deprivation. Semin Neurol. 2005;25(1):117-129.

4. Millman RP; Working Group on Sleepiness in Adolescents/Young Adults; AAP Committee on Adolescence. Excessive sleepiness in adolescents and young adults: causes, consequences, and treatment strategies. Pediatrics. 2005;115(6):1774-1786.

5. Carskadon MA. Patterns of sleep and sleepiness in adolescents. Pediatrician. 1990;17(1):5-12.

6. Carskadon MA, Vieira C, Acebo C. Association between puberty and delayed phase preference. Sleep. 1993;16(3):258-262.

7. Valdez P, Ramirez C, Garcia A. Delaying and extending sleep during weekends: sleep recovery or circadian effect? Chronobiol Int. 1996; 13(3):191-198.

8. Blissitt PA. Sleep, memory, and learning. J Neurosci Nurs. 2001;33(4): 208-218. 
9. McCoy JG, Strecker RE. The cognitive cost of sleep loss. Neurobiol Learn Mem. 2011;96(4):564-582.

10. Born J, Rasch B, Gais S. Sleep to remember. Neuroscientist. 2006;12(5): $410-424$.

11. Heller HC, Ruby NF, Rolls A, Makam M, Colas D. Adaptive and pathological inhibition of neuroplasticity associated with circadian rhythms and sleep. Behav Neurosci. 2014;128(3):273-282.

12. Singleton RA, Wolfson AR. Alcohol consumption, sleep and academic performance among college students. J Stud Alcohol Drugs. 2009;70(3):355-366.

13. Kelly WE, Kelly KE, Clanton RC. The relationship between sleep length and grade point average among college students. Coll Stud J. 2001;35:84-86.

14. Buboltz W, Brown F, Soper B. Sleep habits and patterns of college students: a preliminary study. J Am Coll Health. 2001;50(3):131-135.

15. Allen-Gomes A, Tavares J, Pinto De Azevedo MH. Sleep-wake patterns in Portuguese undergraduates. Acta Med Port. 2009;22(5):545-552.

16. Canet T. Sleep-wake habits in Spanish primary school children. Sleep Med. 2010;11(9):917-921.

17. Park YM, Matsumoto K, Shinkoda H, Nagashima H, Kang MJ, Seo YJ. Age and gender difference in habitual sleep-wake rhythm. Psychiatry Clin Neurosci. 2001;55(3):201-202.

18. Lindberg E, Janson C, Gislason T, Bjornsson E, Hetta J, Boman G. Sleep disturbances in a young adult population: can gender differences be explained by differences in psychological status? Sleep. 1997;20(6): 381-387.

19. Reyner LA, Horne JA, Reyner A. Gender- and age-related differences in sleep determined by home-recorded sleep logs and actimetry from 400 adults. Sleep. 1995;18(2):127-134.

20. Doi Y, Minowa M, Uchiyama M, Okawa M. Subjective sleep quality and sleep problems in the general Japanese adult population. Psychiatry Clin Neurosci. 2001;55(3):213-215.

21. Armitage R, Hoffmann RF. Sleep EEG, depression and gender. Sleep Med Rev. 2001;5(3):237-246.

22. Kessler RC. Gender differences in major depression. Epidemiological findings. In: Frank E, editor. Gender and Its Effects on Psychopathology. Washington, DC: American Psychiatric Press; 2000:61-84.

23. Nolen-Hoeksema S. Gender differences in depression. Curr Dir Psychol Sci. 2001;10(5):173-176.

24. Piccinelli M, Wilkinson G. Gender differences in depression. $\mathrm{Br} J$ Pshychiatry. 2000;177:486-492.

25. Tanios CY, Abou-Saleh MT, Karam AN, Salamoun MM, Mneimneh ZN, Karam EG. The epidemiology of anxiety disorders in the Arab World: a review. J Anxiety Disord. 2009;23(4):409-419.

26. Al-Krenawi A, Lev-Wiesel R, Sehwail MA. Psychological symptomology among Palestinian male and female adolescents living under political violence 2004-2005. Community Ment Health J. 2007;43(1):49-56.

27. El-Khoury W, Kosremelli-Asmar M, Stephan-Yeretzian J, et al. National health statistics report in Lebanon. Institute of Health Management and Social Protection. 2012; pp. 58-62.
28. Karam EG, Mneimneh ZN, Dimassi H, et al. Lifetime prevalence of mental disorders in Lebanon: first onset, treatment, and exposure to war. PLoS Med. 2008;5(4):579-586.

29. Sweileh WM, Ali IA, Sawalha AF, Abu-Taha AS, Zyoud SH, Al-Jabi SW. Gender differences in sleep habits and sleep-related problems in Arab Palestinian university students. Int J Disabil Hum Dev. 2012;11:289-293.

30. Kabrita CS, Hajjar-Muca T, Duffy JF. Predictors of poor sleep quality among Lebanese university students: association between evening typology, lifestyle behaviors, and sleep habits. Nat Sci Sleep. 2014;6:11-18.

31. Buysse DJ, Reynolds CF 3rd, Monk TH, Berman SR, Kupfer DJ. The Pittsburgh Sleep Quality Index: a new instrument for psychiatric practice and research. Psychiatry Res. 1989;28(2):193-213.

32. Zich JM, Attkisson CC, Greenfield TK. Screening for depression in primary care clinics: the CES-D and the BDI. Int J Psychiatry Med. 1990;20(3):259-277.

33. Ensel W. Measuring depression: the CES-D Scale. In: Lin N, Dean A, Ensel WM, editors. Social Support, Life Events and Depression. New york: Academic Press; 1986:55-70.

34. Lund HG, Reider BD, Whiting AB, Prichard JR. Sleep patterns of predictors of disturbed sleep in a large population of college students. J Adolesc Health. 2010;46(2):124-132.

35. Suen LK, Ellis Hon LK, Tam WW. Association between sleep behavior and sleep-related factors among university students in Hong Kong. Chronobiol Int. 2008;25(5):760-775.

36. Sweileh WM, Ali IA, Sawalha AF, Abu-Taha AS, Zyoud SH, Al-Jabi SW. Sleep habits and sleep problems among Palestinian students. Child Adolesc Psychiatry Ment Health. 2011;5(1):25-33.

37. Janson C, Gislason T, De Backer W, et al. Prevalence of sleep disturbances among young adults in three European countries. Sleep. 1995;18(7):589-597.

38. Tsai LL, Li SP. Sleep patterns in college students: gender and grade differences. J Psychosom Res. 2004;56(2):231-237.

39. Burgess HJ, Eastman CI. A late wake time phase delays the human dim light melatonin rhythm. Neurosci Lett. 2006;395(3):191-195.

40. Banser M, Fomberstein KM, Razavi FM, et al. American time use survey: sleep time and its relationship to waking activities. Sleep. 2007;30(9):1085-1095.

41. Matsushita M, Adachi H, Arakida M, et al. Presenteeism in college students: reliability and validity of the Presenteeism Scale for students. Qual Life Res. 2011;20(3):439-446.

42. Erdem C, Senturk I, Arslan CK. Factors affecting grade point average of university students. Empir Econ Lett. 2007;6(5):359-368.

43. Hoskins S, Newstead S, Dennis I. Degree performance as a function of age, gender, prior qualifications and discipline studied. Assess Eval High Educ. 1997;22(3):317-328.

44. Mellanby J, Martin M, O'Doherty J. The 'gender gap' in final examination results at Oxford University. Br J Psychol. 2000;91(pt 3): $377-390$
Nature and Science of Sleep

\section{Publish your work in this journal}

Nature and Science of Sleep is an international, peer-reviewed, open access journal covering all aspects of sleep science and sleep medicine, including the neurophysiology and functions of sleep, the genetics of sleep, sleep and society, biological rhythms, dreaming, sleep disorders and therapy, and strategies to optimize healthy sleep. The manuscript
Dovepress

management system is completely online and includes a very quick and fair peer-review system, which is all easy to use. Visit http://www dovepress.com/testimonials.php to read real quotes from published authors. 